\title{
Riding the Tide Toward the Digital Era: The Imminent Alliance of Al and Mental Health in the Philippines
}

Shaira L. Kee ${ }^{1,2}$, Nicholle Mae Amor T. Maravilla ${ }^{2}$, J ohn Patrick Garganera ${ }^{3}$, Wilbert Garganera ${ }^{4}$, J amie L. Fermin ${ }^{2,5}$, Nouar AIDahoul ${ }^{6}$, Hezerul A. Karim ${ }^{6 *}$, Myles J oshua T. Tan ${ }^{1,2,}$ 7*

${ }^{1}$ Department of Natural Sciences, University of St. La Salle, Philippines, ${ }^{2}$ Yo-Vivo Corporation, Philippines, ${ }^{3}$ University of the East Ramon Magsaysay Memorial Medical Center, Philippines, ${ }^{4}$ Department of Mathematics, Ateneo de Manila University, Philippines, ${ }^{5}$ Department of Electronics Engineering, University of St. La Salle, Philippines, ${ }^{6}$ Faculty of Engineering, Multimedia University, Malaysia, ${ }^{7}$ Department of Chemical Engineering, University of St. La Salle, Philippines

Submitted to J ournal:

Frontiers in Psychiatry

Specialty Section:

Digital Mental Health

Article type:

Opinion Article

Manuscript ID:

746906

Received on:

25 J ul 2021

Journal website link:

www. frontiersin.org 


\section{Conflict of interest statement}

\section{The authors declare a potential conflict of interest and state it below}

S.L.K., N.M.A.T.M., J.L.F., N.A., and M.J.T.T. are affiliated with Yo-Vivo Corporation, a telemedicine service provider based in the Philippines. Yo-Vivo Corporation did not financially support this work.

\section{Author contribution statement}

Conceived the work: S.L.K., N.M.A.T.M., J.L.F., H.A.K., M.J .T.T.; Drafted the article: S.L.K., N.M.A.T.M., J.P.G., W.G.; Critically revised the manuscript: J.L.F., N.A., H.A.K., M.J.T.T.; Acquired funding: H.A.K. All authors contributed to the article and approved the submitted version.

\section{Keywords}

artificial intelligence, machine learning, cognitive behavioral therapy, Biomedical Engineering, Mental Health, Philippines, Psychiatry, healthcare

\section{Contribution to the field}

From a public health perspective, this opinion article discusses why it is necessary to integrate Artificial Intelligence (Al) into the mental health practices in the Philippines. The use of Al systems is an optimum solution to the rising demand for more accessible, cost-efficient, and inclusive healthcare. With the recent developments, the Philippines is deemed to have sufficient capacity to adopt this agendum. This article serves as a call for the introduction of advanced detection tools and predictive analytics in the medical field, especially in the mental health discipline.

\section{Funding statement}

The publication of this article was funded by Multimedia University, Malaysia. 


\section{Riding the Tide Toward the Digital Era: The Imminent Alliance of Al and Mental Health in the Philippines}

Shaira Limson $\mathrm{Kee}^{1,2}$, Nicholle Mae Amor Tan Maravilla ${ }^{2}$, John Patrick Garganera $^{3}$, Wilbert Garganera 4 , Jamie Ledesma Fermin ${ }^{2,5}$, Nouar AlDahoul $^{2,6}$, Hezerul Abdul Karim ${ }^{6 *}$, Myles Joshua Toledo Tan ${ }^{1,2,7 *}$

†These authors have contributed equally to this work and share first authorship. ${ }^{1}$ Department of Natural Sciences, University of St. La Salle, Bacolod, Philippines ${ }^{2}$ Yo-Vivo Corporation, Bacolod City, Philippines

${ }^{3}$ University of the East Ramon Magsaysay Memorial Medical Center, Quezon, Philippines ${ }^{4}$ Department of Mathematics, Ateneo de Manila University, Quezon, Philippines ${ }^{5}$ Department of Electronics Engineering, University of St. La Salle, Bacolod, Philippines ${ }^{6}$ Faculty of Engineering, Multimedia University, Cyberjaya, Malaysia

${ }^{7}$ Department of Chemical Engineering, University of St. La Salle, Bacolod, Philippines

* Correspondence:

Hezerul Abdul Karim

hezerul@mmu.edu.my

Myles Joshua Toledo Tan

mj.tan@usls.edu.ph

Keywords: artificial intelligence, machine learning, cognitive behavioral therapy, biomedical engineering, mental health, Philippines, psychiatry, healthcare

\section{Introduction}

Although the Philippines is a developing country, digital technologies, including the internet and smartphones, are widely used for social communication, entertainment, and dissemination of information (Toquero and Talidong, 2020). 
As of 2021, the current population of the Philippines stands at 110.3 million, making it the 13th most populated country in the world. Of this population, 73.9 million are internet users, and 89.0 million are social media users. Moreover, there are 152.4 million reported mobile connections in the country (Datareportal, 2021). ${ }^{1}$ These are higher compared with data from 2011 when, of over 94 million population, 29.7 million were internet users, 26.7 million were social media users, and 88.1 million were mobile subscribers (Datareportal, 2011).

The high prevalence of digital technology in the country may be due to the fact that online interactions, such as text-based relationships, are psychologically rewarding for some individuals (Griffiths, 2000). For instance, social media platforms, such as Facebook and Twitter, are frequently used by Filipinos to build social relationships in the virtual world and many times, to temporarily divert their attention from the stressful life events that they experience (Toquero and Talidong, 2020). However, these interactions supply the individual with quick doses of dopamine which can later lead to addiction. Individuals with internet addiction show excessive or poorly controlled use of gadgets and the internet, which leads to behavioral or psychological problems (Shaw and Black, 2008; Tran et al, 2017). To prevent this, Artificial Intelligence (AI) can be incorporated into digital technologies by using behavior therapy and activity scheduling to reestablish daily routines, thereby improving the wellbeing of individuals (Cecula, 2021; Tran et al, 2017).

The growth of digital technologies in the country presents a major opportunity to increase mental healthcare. AI technologies can aid in psychiatry education, psychological assessment, diagnosis, and treatment (Luxton, 2014). Machine learning (ML), a branch of AI, can also be utilized in making risk prediction models to estimate the likelihood of developing mental health problems (Shatte et al, 2019). While new technologies, including AI, have already contributed to the various facets of healthcare in other countries (World Health Organization, 2021), healthcare services, most especially mental healthcare, are still outdated and lacking in the Philippines (Maravilla and Tan, 2021). To address this problem, we hope to encourage discussions that will promote the use of $\mathrm{AI}$ in enhancing the mental health of every Filipino.

\section{Predictive Analytics and Detection}

While AI is becoming more popular in the clinical setting, mainstream adoption in healthcare is still far from reach, with the mental health discipline being slower to adopt this technology (Miller and Brown, 2018). AI is an efficient tool for facilitating diagnosis and predicting disease progression, treatment patterns, and drug effects and interactions (Jones et al., 2018). This technology is excellent in synthesizing extensive amounts of information, thereby producing computations that unveil

\footnotetext{
${ }^{1}$ Note: Individuals may have more than one mobile connection, so the number of mobile connections may exceed the total population.
} 
trends and patterns in behavior instantaneously, a task that is often difficult for humans. For this reason, the primary strength of AI is its expeditious pattern analysis (Graham et al., 2019).

The use of ML algorithms such as decision trees, naïve Bayes classifiers, K-nearest neighbor classifiers, logistic regression, deep neural networks, and support vector machines (SVM) enables the accurate prediction of an individual's mental health status. SVMs are the most prominent of these techniques, especially in neuroimaging literature (Jiang et al., 2017; Vapnik, 2000). In fact, SVM has been used to examine the diagnostic value of structural MRI and has been able to discriminate between schizophrenia and healthy controls with an accuracy of 81.1\% (Davatzikos, 2005). These ML techniques can be summarized into three common learning styles: supervised, unsupervised, and deep learning (Wiens \& Shenoy, 2017).

Furthermore, Natural Language Processing (NLP) is also a subfield of AI that focuses explicitly on the analysis of human language. Since most clinical documentation is structured as text, the practice significantly relies on NLP (Névéol et al., 2018). Mindcoa.ch, a mobile health platform that provides access to mental support systems, is powered by IBM ${ }^{\circledR}$ Watson ${ }^{\mathrm{TM}}$ AI, which uses NLP and ML (Beer, 2018). Woebot $^{\mathrm{TM}}$ is another mobile-based conversational agent that aids in monitoring symptoms of depression and anxiety by simulating human support (Fitzpatrick et al., 2017). These conversational agents challenge the perception that digital therapeutics are ineffective in establishing therapeutic relationships with its users (Darcy et al., 2021).

These systems are a leap toward more inclusive and personalized medicine (Darcy et al., 2021; Somashekhar et al., 2017). Depression, schizophrenia, and suicidal ideation are among the mental illnesses that can be predicted using variables like Electronic Health Records, mood rating scales, brain imaging data, novel monitoring systems, and social media platforms (Graham et al., 2019).

These algorithmic methods are fundamental in improving efficiency in the healthcare sector, particularly in the Philippines. The country still faces a crisis because of the predominance of conventional statistical approaches, mapping software, and paper-based reporting systems, as presented by the Philippine Integrated Disease Surveillance and Response manual of the Department of Health (DOH) (DOH, 2017). These traditional and outdated systems hinder the adoption of AI on a national scale, as the size and quality of data can potentially limit the performance of any AI technique.

\section{Digital Mental Health Interventions}

With AI advancements, numerous Digital Mental Health Interventions (DMHI) can now be delivered via the internet and mobile applications in response to individuals' 
psychiatric and psychosocial needs (Mohr et al, 2018). Technological devices such as smartphones and personal computers can now supplement Cognitive Behavioral Therapy (CBT), a psychotherapy, typically provided by human therapists in clinics, that improves the functioning and well-being of mentally ill individuals (American Psychiatric Association, 2017; Toquero and Talidong, 2020).

Application-based smartphone interventions like chatbots have been developed based on CBT using NLP to engage with users and promote behavioral activation with mindfulness in order to reduce symptoms of debilitating psychological problems (Wilheim et al, 2020; Vaidyam et al, 2019; Firth et al, 2017; Linardon et al, 2019). Moreover, mental health problems or psychotic behaviors caused by chronic insomnia (Freeman et al, 2017) can now be treated with Sleepio, an evidence-based intervention that uses CBT. Also, reSET, a prescription-based digital treatment approved by the Food and Drug Administration (FDA), has been developed to treat substance abuse disorder, post-traumatic stress disorder, anxiety disorder, and schizophrenia (Weir, 2018).

A new approach to reducing auditory hallucinations in patients with psychotic conditions, called AVATAR therapy, has been developed, as well (Craig et al, 2017). It involves a three-way conversation between a therapist, a patient, and an avatar. The avatar uses a visual representation and speech transformation software that changes the therapist's voice into a voice that closely resembles the pitch and tone of the patient's hallucinated voices. This therapy encourages patients to confront their auditory hallucinations through a dialogue set by the therapist (Torous et al, 2019).

Additionally, there are individuals who strive to overcome mental health problems caused by real-life situations that trouble them. The most successful intervention for this is Virtual Reality (VR), a computer-generated simulation of a three-dimensional image or environment using evidence-based psychological treatments, that trains individuals to respond appropriately to difficult situations. A VR system is designed based on the theoretical understanding of a specific disorder, and is repeatedly given to patients until the right responses are learned. Once learning is successful in the simulated world, patients can then apply their learnings in the real world (Luxton, 2016; Freeman et al, 2017).

All of these AI technologies for mental health can offer more personalized care, access, and support to millions of Filipinos affected by mental health conditions, in a more targeted manner. Because of the lack of mental health facilities and practitioners in the country (Maravilla and Tan, 2021), it may be best to turn to DMHI.

\section{Implications and Considerations}


Despite some efforts to develop AI technologies in the Philippines over the past few years, the nation is still considered a newcomer to the global AI community. To improve the landscape of emerging technologies, including AI, in the country, the Department of Science and Technology (DOST) put the Harmonized National R\&D Agenda 2017-2022 into effect. This declared 2018 as the year when much attention was given to strengthening AI (DOST, 2018). Through the efforts of the agency, the Philippines ranked second among countries that overperformed in ICT areas, as reported by the United Nations Conference on Trade and Development (UNCTAD) Technology and Innovation Report (TIR) 2021. Also according to the TIR 2021, the Philippines is among a handful of developing nations that are particularly inclined to adopt emerging technologies, such as AI, internet of things and big data. Despite the skills gap that exists in the country, the Department of Information and Communications Technology (DICT) has set several programs in motion to address it. These programs, such as the ICT Academy Project under the Digital Workforce Initiative, are aimed primarily at IT-enabled sectors. The DICT has also created the National Broadband Plan, "Free Wi-Fi for All," and the Common Tower Initiative to improve the coverage of high-quality internet connections across the country. Companies in the private sectors are also seen to be very likely to invest in the development of AI in the country as no stringent regulations to AI have been set in place at the moment (Concepcion et al., 2019). We also believe that integrating AI systems into Philippine telehealth is certainly possible since both have an identical goal - to create more accessible healthcare services.

In order to fully obtain the benefits of AI for mental health, ethical issues should be addressed, as well. Clear laws and policies on the use of AI should be prioritized by those who fund, design, regulate, or use AI for mental health, in order to protect the rights of those who seek professional care. In addition, mental healthcare providers must have a full understanding of AI systems that they employ to ensure safety and reliability. They must also have the appropriate education and training to use and maintain these systems. Furthermore, mental health data should always remain confidential. Thus, developers must construct and enforce robust cybersecurity systems for the protection of mental health providers and patients alike. Moreover, AI systems should be designed to fit the socioeconomics of the country, as AI systems developed in high-income economies may not address the needs of developing economies, such as the Philippines. We must also ensure that the AI technologies that we develop for the provision of mental healthcare are devoid of biases based on race, ethnicity, age, religion, language, and gender.

\section{Discussion}

As we, human beings, evolve, AI evolves too. However, unlike AI, we cease to exist without human interactions. When we feel misunderstood and isolated from the real world, we try to divert our attentions and emotions toward the virtual world, instead of seeking professional help. This manner of thinking is due to digital technologies 
being more accessible, cost-efficient, and capable of supporting us without any prejudice. AI specifically designed to treat the mentally ill may soon become comforters of the human race. However, AI is not developed to replace the human touch, but to aid in the provision of mental healthcare, especially in times of crisis. This is especially true in the Philippine setting, where mental health workers and services are hardly available despite the high prevalence of mental illnesses and suicide rates.

Given the various technological advancements in AI designed for mental health and the growth of digital technologies in the Philippines, indeed, many opportunities lie ahead for this country. However, much work needs to be done to attain this. As such, the following prescription is presented to promote AI for the improvement of mental health services in the Philippines.

First, AI courses should be offered in engineering undergraduate and postgraduate programs. Students in medical fields should also be introduced to the potential of AI for use in their future practice. AI courses could also be advantageous in the field of biomedical engineering (BME). In 2020, Fermin and Tan found strong correlations between BME research and various indicators of overall health in Southeast Asian countries. For this reason, BME could contribute greatly to the development of solutions to pressing mental health issues that exist among Filipinos.

Second, predictive analytics and detection tools based on AI for mental health and digital mental health interventions should be introduced to mental health professionals in hospitals and clinics. Considering that many healthcare professionals are unfamiliar with the new methods, regular training should also be provided. Through continuing education, they would be able to provide mental health services that fully satisfy patients' needs. They would also be able to guide their patients in using these new technologies and potentially solidify the trust and relationship between patient and provider.

Lastly, AI for mental health would not be possible without medical and scientific research. To make this possible, increased funding for and investments in research should be made available to accelerate the development of technologies that would support mental health. Furthermore, collaborations and partnerships with foreign research institutions could help local researchers acquire new knowledge and skills in evolving technologies from other countries.

Through these efforts, the Philippines would be able to gradually redefine mental health practices and make them more efficient, inclusive, and accessible. Integrating AI systems and digital tools into the medical field is only befitting in this fast-paced world. With proper judgment, the country could be more than capable to implement these vital changes. It is certainly time to upscale the country's healthcare system if we wish for a society that is on par with developed nations. 


\section{Conflict of Interest}

S.L.K., N.M.A.T.M., J.L.F., N.A., and M.J.T.T. are affiliated with Yo-Vivo Corporation, a telemedicine service provider based in the Philippines. Yo-Vivo Corporation did not financially support this work.

\section{Author Contributions}

Conceived the work: S.L.K., N.M.A.T.M., J.L.F., H.A.K., M.J.T.T.; Drafted the article: S.L.K., N.M.A.T.M., J.P.G., W.G.; Critically revised the manuscript: J.L.F., N.A., H.A.K., M.J.T.T.; Acquired funding: H.A.K. All authors contributed to the article and approved the submitted version.

\section{Funding}

The publication of this article was funded by Multimedia University, Malaysia.

\section{Contribution to the field}

From a public health perspective, this opinion article discusses why it is necessary to integrate Artificial Intelligence (AI) in the mental health practices in the Philippines. The use of AI systems is an optimum solution to the rising demand for more accessible, cost-efficient, and inclusive healthcare. With the recent developments, the Philippines is deemed to have sufficient capacity to adopt this agendum. This article serves as a call for the introduction of advanced detection tools and predictive analytics in the medical field, especially in the mental health discipline.

\section{References}

American Psychiatric Association (2017). What is Cognitive Behavioral Therapy? PTSD Clinical Practice Guideline https://www.apa.org/ptsd-guideline/patients-and-families/cognitive-behavioral.pdf.

Beer, M. (2018). Watson AI powers mindcoa.ch online psychological self-help solution. Available online at:

https://www.ibm.com/blogs/cloud-computing/2018/11/26/watson-ai-mindcoa-ch-psychologica l-help/. Accessed on: July 6, 2021.

Cecula, P., Yu, J., Dawoodbhoy, F.M., Delaney, J., Tan, J., Peacock, I., Cox, B. (2021). Applications of artificial intelligence to improve patient flow on mental health inpatient units Narrative literature review. Heliyon 7 (4), https://doi.org/10.1016/j.heliyon.2021.e06626.

Concepcion, R. S., Bedruz, R. A. R., Culaba, A. B., Dadios, E. P., \& Pascua, A. R. A. R. (2019). The Technology Adoption and Governance of Artificial Intelligence in the Philippines. 2019 IEEE 11th International Conference on Humanoid, Nanotechnology, Information Technology, Communication and Control, Environment, and Management ( HNICEM ) pp 1-10. doi:10.1109/hnicem48295.2019.9072725

Craig, T., Rus-Calafell, M., Ward, T., Leff, J., Huckvale, M., and Howarth, E., et al (2017). AVATAR therapy for auditory verbal hallucinations in people with psychosis: a single-blind, 
randomised controlled trial. Lancet Psychiatry 5:31-40.

http://dx.doi.org/10.1016/S2215-0366(17)30427-3

Darcy A, Daniels J, Salinger D, Wicks P, Robinson A.(2021) Evidence of Human-Level Bonds Established With a Digital Conversational Agent: Cross-sectional, Retrospective Observational Study. JMIR Form Res. 5(5):e27868. doi: 10.2196/27868.

Datareportal (2011). Digital 2011: The Philippines. Available online at: https://datareportal.com/reports/digital-2011-philippines?rq=philippines. Accessed on: July 14, 2021.

Datareportal (2021). Digital 2021: The Philippines. Available online at: https://datareportal.com/reports/digital-2021-philippines?rq=philippines. Accessed on: July 14, 2021.

Davatzikos, C., Ruparel, K., Fan, Y., Shen, D.G., Acharyya, M., Loughead, J.W., Gur, R.C., Langleben, D.D. (2005). Classifying spatial patterns of brain activity with machine learning methods: application to lie detection. Neuroimage 28 (3): 663-668. doi:

10.1016/j.neuroimage.2005.08.009

Department of Health (2017). Manual of Procedures for the Philippine Integrated Disease Surveillance and Response (PIDSR) 3rd Edition (2014). Available online at: https://doh.gov.ph/node/9985. Accessed on: July 6, 2021.

Department of Science and Technology. (2018). Harmonized National R \& D Agenda 2017-2022. Available online at:

https://www.dost.gov.ph/knowledge-resources/downloads/file/791-harmonized-national-r-d-ag enda-2017-2022.html. Accessed on: July 14, 2021.

Fermin, J. and Tan, M.J. (2020). The Need for the Establishment of Biomedical Engineering as an Academic and Professional Discipline in the Philippines-A Quantitative Argument. IEEE Access, 9: 3097-3111. Doi: 10.1109/ACCESS.2020.3046655

Firth, J., Torus, J., Nicholas, J., Carney, R., Pratap, A., Rosenbaum, S., Sarris, J. (2017). The efficacy of smartphone-based mental health interventions for depressive symptoms: a meta-analysis of randomized controlled trials. World Psychiatry 16:287-298. doi:10.1002/wps.20472.

Fitzpatrick, K., Darcy, A.M., \& Vierhile, M. (2017). Delivering Cognitive Behavior Therapy to Young Adults With Symptoms of Depression and Anxiety Using a Fully Automated Conversational Agent (Woebot): A Randomized Controlled Trial. JMIR Mental Health, 4.doi:10.2196/mental.7785

Freeman, D., Sheaves, B., Goodwin, M., Yu, L., Nickless, A., and Harrison, P.J., et al (2017). The effects of improving sleep on mental health (OASIS): a randomised controlled trial with mediation analysis. Lancet Psychiatry 4: 749-58. http://dx.doi.org/10.1016/S2215-0366(17)30328-o

Freeman, D., Reeve, S., Robinson, A., Ehlers, A., Clark, D., Spanlang, B., and Slater, M. (2017). Virtual reality in the assessment, understanding, and treatment of mental health disorders. Psychological Medicine 47(14): 2393-2400. doi:10.1017/So03329171700040X 
Graham, S., Depp, C., Lee, E., Nebeker, C., Tu, X., Kim, H-C., Jeste, D. (2019). Artificial Intelligence for Mental Health and Mental Illnesses: an Overview. Current Psychiatry Reports, 21(11):116. DOI: 10.1007/s11920-019-1094-0.

Griffiths, M. (2000). Does Internet and Computer “Addiction” Exist? Some Case Study Evidence. CyberPsychology \& Behavior 211-218 http://doi.org/10.1089/109493100316067.

Jiang, F., Jiang, Y., Zhi, H., Dong, Y., Li,H., Ma, S., Wang, Y, et al. (2017). Artificial intelligence in healthcare: past, present and future. Stroke and Vascular Neurology 2. doi:

10.1136/svn-2017-000101

Jones, L.D., Golan, D., Hanna, S.A., Ramachandran, M. (2018). Artificial intelligence, machine learning and the evolution of healthcare: A bright future or cause for concern? Bone Joint Res, 7:223-225. DOI: 10.1302/2046-3758.73.BJR-2017-0147.R1

Linardon, J., Cuijpers, P., Carlbring, P., Messer, M., and Fuller-Tyszkiewicz, M. (2019). The efficacy of app-supported smartphone interventions for mental health problems: a meta-analysis of randomized controlled trials. World Psychiatry 18:325-336. doi: 10.1002/wps.20673.

Luxton, D. (2014). Artificial intelligence in psychological practice: Current and future applications and implications. Professional Psychology: Research and Practice 45(5): 332-339. https://doi.org/10.1037/aoo34559

Luxton, D. (2016). Artificial Intelligence in Behavioral and Mental Health Care. Department of Psychiatry and Behavioral Sciences. Washington and California, USA. pp. 1-15.

Maravilla, N.M.A. and Tan, M.J. (2021). Philippine Mental Health Act: Just an Act? A Call to Look Into the Bi-directionality of Mental Health and Economy. Frontiers in Psychology, 12 : 2928. DOI=10.3389/fpsyg.2021.706483

Miller, D.D., Facp, C.M., Brown, E.W.(2018) Artificial intelligence in medical practice: the question to the answer ?. Am J Med., 131(2): 129-33. doi: 10.1016/j.amjmed.2017.10.035.

Mohr, D.C., Riper, H., \& Schueller, S.M. (2018) A Solution-Focused Research Approach to Achieve an Implementable Revolution in Digital Mental Health. JAMA Psychiatry, 75(2):113-114. doi:10.1001/jamapsychiatry.2017.3838

Névéol, A., Dalianis, H., Velupillai, S., Savova, G. and Zweigenbaum, P. (2018). Clinical Natural Language Processing in languages other than English: opportunities and challenges. Journal of Biomedical Semantics 9:12. https://doi.org/10.1186/s13326-018-0179-8

Shatte, A., Hutchinson, D. M., \& Teague, S. J. (2019). Machine learning in mental health: a scoping review of methods and applications. Psychological medicine 49(9): 1426-1448. https://doi.org/10.1017/Soo33291719000151

Shaw, M., and Black, D.W. (2008). Internet addiction: definition, assessment, epidemiology and clinical management. CNS Drugs, 22(5):353-65. doi: 10.2165/00023210-200822050-00001.

Somashekhar, S.P., Kumarc, R., Rauthan, A., Arun, K.R., Patil, P., Ramya, Y.E.(2017). Double blinded validation study to assess performance of IBM artificial intelligence platform, Watson for oncology in comparison with Manipal multidisciplinary tumour board - First study of 638 breast 
cancer cases [abstract]. Cancer Res 77(4 Suppl):Abstract nr S6-07.doi: 10.1158/1538-7445.SABCS16-S6-07

Torous, J., Andersson, G., Bertagnoli, A., Christensen, H. Cuijpers, P. et al (2019). AVATAR therapy: a promising new approach for persistent distressing voices. World Psychiatry 18:1. https://www.ncbi.nlm.nih.gov/pmc/articles/PMC6313231/pdf/WPS-18-97.pdf

Toquero, C., and Talidong, K. (2021). Socio-educational implications of technology use during COVID-19: A case study in General Santos City, Philippines. Human Behavior and Emerging Technologies 3:194 - 198. doi: 10.1002/hbe2.214.

Tran, B.X., Huong, L.T., Hinh, N.D., Nguyen, L.H., Le, B.N. et al. (2017). A study on the influence of internet addiction and online interpersonal influences on health-related quality of life in young Vietnamese. BMC Public Health 17 (138). https://doi.org/10.1186/s12889-016-3983-z

Vaidyam, A.N., Wisniewski, H., Halamka, J.D., Kashavan, M., and Torous, J.B. (2019). Chatbots and Conversational Agents in Mental Health: A Review of the Psychiatric Landscape. The Canadian Journal of Psychiatry 64 (7): 456 - 464. doi: 10.1177/0706743719828977.

Vapnik, V.N. (2000). The Nature of Statistical Learning Theory. New York: Springer. https://doi.org/10.1007/978-1-4757-3264-1

Weir, K. (2018). The ascent of digital therapies. American Psychiatric Association 49 (10): 80. https://www.apa.org/monitor/2018/11/cover-digital-therapies

Wiens, J., Shenoy, E.S. (2018). Machine Learning for Healthcare: On the Verge of a Major Shift in Healthcare Epidemiology. Clinical Infectious Diseases, 66(1):149-153. doi: 10.1093/cid/cix731.

Wilheim, S., Weingarden, H., Ladis, I., Braddick, V., Shin, J. and Jaconson, N. (2020). Cognitive Behavioral Therapy in the Digital Age: Presidential Address. Behavior Therapy 51(1): 1-14. https://doi.org/10.1016/j.beth.2019.08.001.

World Health Organization (2021). Ethics and governance of artificial intelligence for health: WHO guidance. Available online at: https://www.who.int/publications/i/item/9789240029200. Accessed on: July 10, 2021. 